\title{
1 Incorporation of data from multiple hypervariable regions when analyzing bacterial 16S rRNA sequencing data
}

3

25

Carli B. Jones ${ }^{1 \rrbracket}$, James R. White ${ }^{2}$, Sarah E. Ernst ${ }^{1}$, Karen S. Sfanos ${ }^{1,3,4, *}$, Lauren B. Peiffer ${ }^{1,5}$ II* $^{*}$

${ }^{1}$ Department of Pathology, Johns Hopkins University School of Medicine, Baltimore, MD, USA

${ }^{2}$ Resphera Biosciences, Baltimore, MD, USA

${ }^{3}$ Deparment of Oncology, Johns Hopkins University School of Medicine, Baltimore, MD, USA

${ }^{4}$ Department of Urology, Johns Hopkins University School of Medicine, Baltimore, MD, USA

${ }^{5}$ Department of Molecular and Comparative Pathobiology, Johns Hopkins University School of Medicine, Baltimore, MD, USA

* Corresponding authors

Email: ksfanos@jhmi.edu (KSS), 1peiffe1@jhmi.edu (LBP)

II These authors contributed equally to this work.

8

9


Short read 16S rRNA amplicon sequencing is a common technique used in microbiome research. However, inaccuracies in estimated bacterial community composition can occur due to amplification bias of the targeted hypervariable region. A potential solution is to sequence and assess multiple hypervariable regions in tandem, yet there is currently no consensus as to the appropriate method for analyzing this data. Additionally, there are many sequence analysis resources for data produced from the Illumina platform, but fewer open-source options available

37 for data from the Ion Torrent platform. Herein, we present an analysis pipeline using an opensource analysis platform that integrates data from multiple hypervariable regions and is compatible with data produced from the Ion Torrent platform. We used the ThermoFisher Ion $16 \mathrm{~S}^{\mathrm{TM}}$ Metagenomics Kit and a mock community of 20 bacterial strains to assess taxonomic

41 classification of amplicons from 6 separate hypervariable regions (V2, V3, V4, V6-7, V8, V9)

42 using our analysis pipeline. We report that different hypervariable regions have different

43 specificities for taxonomic classification, which also had implications for global level analyses

44 such as alpha and beta diversity. Finally, we utilize a generalized linear modeling approach to

45 statistically integrate the results from multiple hypervariable regions and apply this methodology

46 to data from a small clinical cohort. We conclude that scrutinizing sequencing results separately

47 by hypervariable region provides a more granular view of the taxonomic classification achieved

48 by each primer set as well as the concordance of results across hypervariable regions. However,

49 the data across all hypervariable regions can be combined using generalized linear models to

50 statistically evaluate overall differences in community structure and relatedness among sample

51 groups. 


\section{Introduction}

Next generation sequencing of microbial DNA has become an important tool used for

54 determining relationships between human-associated microbial populations and various diseases.

55 Most studies in this realm rely on either shotgun metagenomic sequencing or 16S ribosomal

56 RNA (rRNA) amplicon sequencing. Shotgun metagenomic sequencing involves sequencing

57 random fragments of sample DNA which contains a mixture of bacterial DNA, as well as host

58 and other microbial and environmental DNA (1). This method allows for taxonomic profiling,

59 metabolic function profiling, and antibiotic resistance gene profiling; however, it is generally

60 more expensive than amplicon sequencing, and requires a large amount of input DNA and the

61 availability of reference genome sequences. Bacterial 16S rRNA amplicon sequencing employs

62 PCR amplification of specific hypervariable regions within the gene, followed by deep

63 sequencing (2). This method is generally a quicker, cheaper alternative to shotgun

64 metagenomics; however, it only identifies bacteria and the typical strategy only sequences a

65 specific fragment of the bacterial 16S rRNA gene (3). The 16S rRNA gene is comprised of 9

hypervariable regions (V1-V9), and most primers used for next generation sequencing only

67 target one to two hypervariable regions at a time. Multiple studies have shown that different

68 regions vary in their taxonomic utility due to a combination of primer bias, differential

69 hypervariable region sequence length, and hypervariable region sequence uniqueness across

70 bacterial taxa (4-8). An ideal solution would be to sequence the entire 16S rRNA gene, however

71 this requires long-read sequencing that is very time and resource intensive. Therefore, a potential

72 alternative would be to perform 16S rRNA amplicon sequencing on multiple regions and

73 incorporate information from as many hypervariable regions as possible into downstream data

74 analysis. 
The Ion $16 \mathrm{~S}^{\mathrm{TM}}$ Metagenomics Kit (Life Technologies) utilizes primers to seven different

76 hypervariable regions: V2, V3, V4, V6-7, V8, and V9. This is an attractive approach because it

77 yields more sequence information across the 16S rRNA gene overall. However, there is currently

78 little consensus as to how to properly analyze information from multiple hypervariable regions

79 and obtain overall results. Current analysis pipelines for Ion Torrent data include the Ion

80 Reporter Software offered by ThermoFisher, and an alternative method using open access tools

81 developed by Barb et al. (5). Both pipelines offer methods for taxonomic identification;

82 however, they do not address the question of how to appropriately integrate data from multiple

83 hypervariable regions in downstream analyses. Recently, Fuks et al. (9) and Debelius et al. (10)

84 developed methods to computationally combine data from multiple hypervariable regions to

85 provide a joint estimate of the microbial community composition. To date, however, there is no

86 generally agreed upon approach for combining sequences from multiple hypervariable regions

87 for downstream analyses, especially for less commonly used 16S rRNA sequencing platforms

88 such as Ion Torrent.

Herein, we developed an analysis pipeline that analyzes data from each hypervariable

90 region separately, allowing for systematic comparison of taxonomic classification by

91 hypervariable region. We demonstrate our results from analyzing a mock community of

92 bacterial DNA where we determine how each hypervariable region differs in its utility to provide

93 information on taxonomic classifications, alpha diversity, and beta diversity. We report that

94 certain taxa are only identified by particular $\mathrm{V}$ regions, supporting our hypothesis that there is a

95 benefit to incorporating multiple primer sets into sequencing strategies. Furthermore, we discuss

96 different options for downstream analysis and statistics, and propose a generalized linear model 
97 (GLM) to statistically combine the results from multiple hypervariable regions. Finally, we

98

99

100

101

102

103

104

105

106

107

108

109

110

111

112

113

114

115

116

117 demonstrate the utility of our approach in the analysis of clinical samples.

\section{Materials and methods}

\section{Clinical sample collection and mock community}

All specimens were studied under an Institutional Review Board (IRB) approved protocol with written informed consent. Three adult males self-collected two rectal swab samples with sterile flocked swabs (Copan Diagnostics, Murrieta, CA). DNA was extracted from one of the swabs from each individual immediately after collection (referred to as RS1). The other swabs (referred to as RS2) were frozen at $-80^{\circ} \mathrm{C}$ for six days before DNA extraction.

The 20 Strain Even Mix Genomic Material was obtained from American Type Culture Collection (ATCC, Cat. No. MSA-1002, Manassas, VA). The strain composition of the mock community is given in Table 1 . The mock community was sequenced with five technical replicates.

\section{DNA extraction}

The DNA extraction protocol was adapted from our previously published protocol (11). Briefly, rectal swab fecal material was resuspended in $500 \mu \mathrm{l}$ of $1 \mathrm{X}$ phosphate buffered saline (PBS). Samples were then digested in a cocktail of lysozyme $(10 \mathrm{mg} / \mathrm{ml}$, Sigma-Aldrich, St. Louis, MO) and mutanolysin $\left(25 \mathrm{KU} / \mathrm{ml}\right.$, Sigma-Aldrich) for 1 hour at $37^{\circ} \mathrm{C}$. The contents of the tubes were then transferred into FastPrep Lysing Matrix B tubes (MP Biomedicals, Santa Ana, CA). Next, 20\% SDS (Sigma-Aldrich) and phenol:chloroform:isoamyl alcohol (25:24:1, ThermoFisher Scientific, Waltham, MA) were added and samples were homogenized by bead 
beating in an MP FastPrep-24 at $6 \mathrm{~m} / \mathrm{s}$ for a total of 60 seconds. DNA was precipitated and resuspended in a final volume of $50 \mu \mathrm{l}$ of DNA-free water (Molzym, Bremen, Germany).

\section{Library preparation}

Concentration of DNA from the mock microbial community (Table 1) and rectal swabs was measured using a Qubit dsDNA HS (high sensitivity) kit (Life Technologies, Carlsbad, CA). Libraries were prepared using the Ion 16S ${ }^{\mathrm{TM}}$ Metagenomics Kit (Cat. No. A26216,

ThermoFisher Scientific). Briefly, $10 \mathrm{ng}$ of DNA was mixed with $15 \mu \mathrm{l}$ of Environmental Master Mix. $3 \mu 1$ of each 16S Primer Set (10X) was added to each tube, one sample set with primers for V2-4-8 (Pool 1) and the other with primers for V3-6,7-9 (Pool 2). Samples were placed in a thermocycler with the following thermal conditions: $95^{\circ} \mathrm{C}$ for 10 minutes, 25 cycles of $95^{\circ} \mathrm{C}$ for $30 \mathrm{~s}, 58^{\circ} \mathrm{C}$ for $30 \mathrm{~s}, 72^{\circ} \mathrm{C}$ for $30 \mathrm{~s}$, then $72^{\circ} \mathrm{C}$ for $7 \mathrm{~min}$. Amplification products were purified using AMPure XP beads (Cat. No. A63881, Beckman Coulter, Pasadena, CA) and eluted in nuclease free water. Concentrations of amplification products from Pool 1 and Pool 2 were measured using a Bioanalyzer and the High Sensitivity DNA Kit (Agilent Technologies, Santa Clara, CA), and the two pools were combined for a total of $100 \mathrm{ng}$ of DNA (50ng from each pool).

Next, $20 \mu \mathrm{l}$ of 5X End Repair Buffer and $1 \mu \mathrm{l}$ of End Repair Enzyme were added to each sample, and then incubated for 20 min at room temperature. Pooled amplicons were then purified again using AMPure XP beads and eluted in Low TE buffer. Ligation and nick repair were performed using 10X Ligase Buffer, Ion P1 Adaptor, Ion Xpress Barcodes, dNTP Mix, DNA Ligase, Nick Repair Polymerase, nuclease-free water, and sample DNA with the following thermal conditions: $25^{\circ} \mathrm{C}$ for $15 \mathrm{~min}, 72^{\circ} \mathrm{C}$ for $5 \mathrm{~min}$. Adapter-ligated and nick-repaired DNA was then purified using AMPure XP beads and eluted in Low TE buffer. 
The library was then amplified using the Ion Plus Fragment Library Kit (Cat. No.

141

142

143

144

145

146 4471252, ThermoFisher Scientific) with the following thermal conditions: $95^{\circ} \mathrm{C}$ for $5 \mathrm{~min}, 7$ cycles of $95^{\circ} \mathrm{C}$ for $15 \mathrm{~s}, 58^{\circ} \mathrm{C}$ for $15 \mathrm{~s}, 70^{\circ} \mathrm{C}$ for $1 \mathrm{~min}$, and then $70^{\circ} \mathrm{C}$ for $1 \mathrm{~min}$. The amplified library was then purified using AMPure XP beads and eluted in Low TE buffer. Library concentrations were measured using a Bioanalyzer and the High Sensitivity DNA Kit. Libraries were then diluted down to $26 \mathrm{pM}$ and pooled, yielding a $26 \mathrm{pM}$ solution.

\section{Sequencing}

Libraries were prepared for sequencing using oil amplification to template the libraries onto beads and loaded onto chips using the Ion Chef Instrument and the Ion $520^{\mathrm{TM}} \&$ Ion $530^{\mathrm{TM}}$ Kit - Chef (ThermoFisher Scientific). Chips were then loaded onto the Ion GeneStudio S5 System along with Ion S5 Sequencing Kit reagents (ThermoFisher Scientific) and sequenced. Samples in this study were sequenced across three separate sequencing runs on Ion 520 and Ion 530 chips using $400 \mathrm{bp}$ sequencing kits. Sequences were demultiplexed by sample using the S5 device software, and then separated per hypervariable region by ThermoFisher prior to downstream analysis.

\section{Database curation}

When using SILVA (12) or Greengenes (13) as our reference database, approximately $50 \%$ of reference sequences were unclassified. Therefore, we utilized a refined version of the SILVA (v.123) database for taxonomic assignment with unclassified and distantly related sequences were removed. The refined database (sfanos- $d b-4.0$ ) contains approximately 15,000 named species and includes species that are of particular interest for future clinical gastrointestinal (GI) microbiome studies. 


\section{2 \\ In silico taxonomic validation of curated database}

To determine how well our curated database could assign taxonomy to sequences and

164 how much the assignment differs by hypervariable region, we created a system of in silico

165 taxonomic validation using a human gut microbiome culture collection (14). First, we separated

166 the sequences in the culture collection by hypervariable region. To do this, we ran the sequences

167 from the culture collection through NCBI BLAST against the ATCC mock community

168 sequences that had already been split by hypervariable region. This method allowed us to break

169 down the culture collection sequences into their different hypervariable regions and simulate

170 more complex clinical data. A $1 \%$ noise rate was included in the simulated sequences to mimic

171 typical evolutionary variation in species as well as sequencing error. We then ran taxonomic

172 classification of the sequences from the culture collection using our curated database, with a

173 threshold of $97 \%$ sequence identity. A confidence score was assigned to each classification by

174 VSEARCH. Results were categorized into true positives, false positives, and false negatives

175 based on whether they were in the culture collection or not.

\section{Data processing}

The ThermoFisher Bioinformatics team divided each fastq file by hypervariable region

178 using proprietary primer sequences to produce 6 separate fastq files per sample (V2, V3, V4, V6-

179 7, V8, and V9), with primer sequences removed and all reads oriented in the forward direction.

180 Manifest files were then created for each hypervariable region and each sequencing run. Fastq

181 files were imported into QIIME2 format via qiime tools import in

182 SingleEndFastqManifestPhred33V2 format (15). QIIME2 v 2020.6 was used to perform 
denoising, Operational Taxonomic Unit (OTU) clustering, taxonomic classification, phylogenetic tree construction, and alpha and beta diversity.

DADA2 was used to denoise data, using the denoise-pyro plugin and parameters of 0bp for trimming and truncation (16). A separate DADA2 run was performed for each hypervariable region and each sequencing run. Denoising statistics were then exported and summarized via the B02-summarize-qc.pl Perl script. From this summary, we determined that all samples in all hypervariable regions had a minimum of 10,000 reads which passed the filter in the DADA2 step. Good's coverage was performed at a depth of 10,000 reads for each hypervariable region and at least $99 \%$ coverage was achieved for all regions (17). Thus, we decided that 10,000 reads was an acceptable sampling depth. DADA2 feature tables and representative sequence files were then merged across sequencing runs so that there was only one feature table and representative sequence file per hypervariable region.

Open-reference OTU clustering was then performed using QIIME2 plugin vsearch cluster-features-open-reference (18). A threshold of 99\% identity was used, and sequences were clustered against reference sequences from the curated sfanos_db_v4.0.fastq.qza database.

\section{Alpha and beta diversity analysis}

A phylogenetic tree was constructed for each hypervariable region using the representative sequences file generated from open-reference OTU clustering via the qiime phylogeny align-to-tree-mafft-fasttree plugin (19-21), Shannon diversity (22), evenness, and observed OTUs. Distance matrices were exported for Jaccard (23), Bray-Curtis (24), weighted (25), and unweighted (26) UNIFRAC distances. Distance matrices were created from filtered taxonomic abundance results comparing region to region. Visualizations of alpha diversity 
metrics as well as principal coordinates analysis (PCoA) plots were created using $\mathrm{R}$ in order to determine region to region differences.

\section{Taxonomic classification}

Taxonomic classification was performed using classify-consensus-vsearch using the curated sfanos_db_v4.0 reference reads and reference taxonomy with 99\% identity. The output .qza file was then exported in order to obtain the taxonomy.tsv file. This file and the featuretable.biom file were used in a Perl script designed to summarize the taxonomic information into

212 feature-table-with-taxonomy.txt. Heatmaps were created in R using the pheatmap package and taxa-normalize-pct-per-region.txt file.

\section{Contaminant filtering}

217 both, was considered a contaminant. Filtering was performed on the feature table that was

218 created after open reference OTU clustering using qiime taxa filter-table. Contaminants are listed 219 in S1 Table.

\section{Generalized linear modeling}

We used the generalized linear model function in Base $\mathrm{R}$ to evaluate statistical

222 differences in alpha diversity and individual taxonomic abundance between fresh versus frozen

223 samples in the clinical cohort. The GLM per feature took the following structure: $\log 10$ (feature)

$224 \sim$ fresh/frozen status + specimen ID + hypervariable region. Regions V8 and V9 were excluded

225 from GLM analysis, and Region V2 was used as the null factor level. The fresh/frozen status of 
samples was compared, with fresh as baseline factor level set as zero and frozen set as one. The input of "feature" was either an alpha diversity value (Shannon, Evenness, Observed OTUs or Faith's Phylogenetic Diversity), or taxonomic abundance of a feature at a specific taxonomic level. Input feature values were log transformed in order to increase stability of values from person to person when performing statistics. The GLM p-value was obtained by comparing the GLM factor level coefficient to the null hypothesis of zero, which was done via a Wald Test.

\section{Data and code availability}

All sequences were submitted to the NCBI Sequence Read Archive (SRA) under Bioproject ID PRJNA738491. Sequences will be released upon publication in a peerreviewed journal. All codes used can be found on the public GitHub repository it-workflow (http://github.com/Sfanos-Lab-Microbiome-Projects/it-workflow/), which will be made public upon publication in a peer-reviewed journal.

\section{Results}

\section{In silico validation of taxonomic classification accuracy of curated database}

We curated a database specific for our needs during our pipeline development using SILVA as the base. We then validated the classification accuracy of this database by performing an in silico analysis where we ran known sequences from a human gut microbiome culture collection (14) through our classification scheme and recorded whether the sequences were classified correctly (true positive), incorrectly (false positive), or not identified (false negative).

Results were compiled by unique sequence identifier in a pivot table which noted the corresponding hypervariable region, true taxonomic classification at the genus and species 
levels, taxonomic classification by VSEARCH using our curated database, VSEARCH confidence score, and classification of assignment by VSEARCH at both the genus and species level as either True Positive (TP), False Positive (FP), or False Negative (FN) (S1 File). sequences for that region, and multiplying by 100. Taxonomic classification across all $\mathrm{V}$ regions was more accurate at the genus level than the species level, with a combined total of $85.08 \% \mathrm{TP}$, $6.95 \% \mathrm{FP}$, and $7.97 \% \mathrm{FN}$ verses $65.96 \% \mathrm{TP}, 10.31 \% \mathrm{FP}$, and $23.74 \% \mathrm{FN}$, respectively. Results were not evenly distributed across V regions. V3 had the highest accuracy at the genus level in all three metrics whereas V2, V4, and V6-7 performed similarly to each other and slightly less accurately than V3. V8 and V9 had lower TP and higher FN and FP than all other V regions counts and highest TP counts. Regions V8 and V9 had much lower accuracy with the highest FP and FN counts and lowest TP counts. Regions V4 and V6-7 performed in between (Table 2).

262

267 diversity and taxonomic abundance analyses (see Methods).

\section{Mock community}

In order to test our analysis pipeline, we prepared libraries and sequenced DNA from a mock microbial community (20 Strain Even Mix Genomic Material, ATCC® MSA-1002 ${ }^{\mathrm{TM}}$ ). Five independent replicate library preparations of the mock community were sequenced. We filtered out low-level contaminants (S1 Table) prior to performing community alpha and beta

\section{Alpha diversity}

We analyzed 4 different alpha diversity metrics, 2 measures of evenness (Evenness and 
271 OTUs) (Fig 1). V9 has significantly decreased alpha diversity compared to all regions across all

272 metrics (S2 File). V8 also has significantly decreased Shannon diversity, Evenness, and Faith’s

273 phylogenetic diversity compared to other regions excluding V9, with two exceptions being that

274 Evenness is not significantly decreased in V8 compared to that of V6-7 and Faith's PD is not

275 significantly decreased in V8 compared to V4 (S2 File).

\section{Beta diversity}

To compare beta diversity between hypervariable regions and circumvent the issue that OTUs would be region-specific, we used taxonomic results from each hypervariable region to create aggregated distance matrices. We assessed six different beta diversity metrics: Canberra, Bray-Curtis, Jaccard, Euclidean, Gower, and Kulczynski. Fig 2 shows a PCoA plot based on the Canberra distance matrix. The V2, V3, V4, and V6-7 hypervariable regions clustered together, whereas V8 and V9 were distantly separated. This pattern was observed by other beta diversity metrics, with V6-7 sometimes also segregating slightly from V2, V3, and V4 which were largely clustered together (S1 Fig).

\section{Taxonomic classification and abundance}

in S3 File. The majority of species were identified by taxonomic classification of the sequences covering each hypervariable region, with the exception of V9 that only positively identified

292 V8 mis-classified it as Clostridium butyricum, and V9 did not identify any Clostridial organisms 
We next compared observed versus expected percent abundance by hypervariable region.

295

296

297

298

299

300

301

302

303

304

305

306

307

To determine the expected percent abundance, we divided the number of 16S rRNA copies

present in each bacterium (Table 1) and divided by the total number of $16 \mathrm{~S}$ rRNA copies in the 20 Strain Even Mix. Taxonomic bar plots demonstrate the percent abundance of each taxa by hypervariable region compared to expected (Fig 3). V2 most closely approximated the overall distribution of species compared to expected and correctly assigned the most species from the mock community (19/20). V3 (17/20), V6-7 (17/20), and V4 (16/20) followed closely behind, whereas V8 assigned 15/20, and V9 was only able to identify two species (2/20) (Table 3).

Lastly, we performed a clustered heatmap analysis at the species level. The resulting heatmap demonstrated that technical replicates of the mock community sequences cluster by hypervariable region (Fig 4). The heatmap visually emphasizes the difference in taxonomic identification in V8 and particularly V9 compared to the other regions. It also highlights misclassifications and which regions were only able to classify taxa to the genus level.

\section{Clinical samples}

We next sequenced and analyzed a set of six clinical samples in order to demonstrate the use of our pipeline on a clinical sample set. Samples consisted of duplicate rectal swabs from 3 participants. DNA was extracted immediately after collection from 1 rectal swab sample from each person (Fresh) and the other sample was frozen at $-80{ }^{\circ} \mathrm{C}$ prior to DNA isolation (Frozen).

Libraries were prepared in tandem, and all samples were sequenced on the same sequencing run. Sequencing results were processed as outlined above.

\section{Alpha diversity}

We performed the same four alpha diversity metrics for the clinical cohort as for the mock community samples (Evenness, Shannon diversity, Observed OTUs, and Faith's phylogenetic diversity). We used a GLM (see Methods) to incorporate data from hypervariable 
regions V2, V3, V4, and V6-7 in statistical analyses. We excluded data from the V8 and V9 regions due to the demonstrated poor performance of amplicons from these regions in identifying species in our in silico analysis and in the mock community (Table 2, Figs 1-4). There were no significant differences in alpha diversity between fresh and frozen samples by Shannon diversity, Faith's phylogenetic diversity or Observed OTUs (Fig 5). Evenness was slightly increased in frozen samples across all hypervariable regions (adjusted GLM $\mathrm{p}=0.015$ ).

\section{Beta diversity}

We aggregated taxonomic results and used them to create Bray-Curtis, Jaccard, Canberra, Euclidean, Gower, and Kulczynski distance matrices in order to perform combined beta diversity analysis across all hypervariable regions. As demonstrated by the Canberra PCoA plot in Fig 6, most variation in Beta diversity was due different individuals and V9 sequences. PERMANOVA analysis of results from each individual hypervariable region demonstrated that total composition does not differ by Fresh versus Frozen status after adjusting for individual person and region-toregion variation (S4 File).

\section{Taxonomic differential abundance}

We systematically compared abundance of taxa between fresh and frozen samples at multiple levels (phylum, class, order, family, genus, species). We specifically looked at levels of Firmicutes, Bacteroidetes, and Faecalibacterium due to previous reports of differential abundance in fresh verses frozen samples $(27,28)$. Our results showed no significant differences in these taxa (Fig 7) or Firmicutes to Bacteroidetes ratios (Fig 8).

\section{Discussion}

$16 \mathrm{~S}$ rRNA sequencing is a popular method for microbiome characterization due to multiple factors. The 16S rRNA gene encodes the small subunit of the prokaryotic ribosome and 
thus is present in the genome of all bacteria. The genetic sequence of $16 \mathrm{~S}$ rRNA is composed of alternating conserved regions and nine hypervariable regions. PCR amplification using primers that target conserved regions and amplify across hypervariable regions allows amplification of DNA across a widespread taxonomic spectrum, and provides unique sequences that can be used for taxonomic classification at higher levels (e.g., family, genus, and species level). Next generation sequencing strategies are often limited to sequencing across only one or at most two

347 of the nine hypervariable regions. The Ion $16 \mathrm{~S}^{\mathrm{TM}}$ Metagenomics Kit provides the opportunity to

348 prepare libraries containing sequences from seven of the nine hypervariable regions (V2, V3, V4, V6-7, V8, and V9). Herein we report results from sequencing a mock microbial community using the Ion $16 \mathrm{~S}^{\mathrm{TM}}$ Metagenomics Kit and comparing results from different hypervariable regions. We also propose using a generalized linear multivariate model to incorporate

352 sequencing data from multiple hypervariable regions for analyzing diversity and taxonomic 353 abundances and demonstrate the use of this model in a small cohort of clinical samples.

355 Greengenes and SILVA databases are commonly used for taxonomic classification, however they are infrequently updated, inconsistently annotated, and contain redundant sequences (12,

357 13). Therefore, we refined the SILVA database by removing unclassified and distantly related 358 sequences and formatting taxonomic annotations into a uniform layout to make sfanos db 4.0.

359 Prior to using our database, we confirmed its accuracy by performing an in silico analysis using a 360 human gut microbiome culture collection (14) where we ran known sequences through our 361 classification scheme and recorded whether the sequences were classified correctly (true 362 positive), incorrectly (false positive), or not identified (false negative). Encouragingly, our database correctly identified $85.05 \%$ of sequences to the genus level and $65.96 \%$ of sequences to 
the species level, which is an improvement compared to the rates previously published for the standard Greenegenes and SILVA databases (29). While our results are encouraging, it is important to keep in mind that nearly $44 \%$ of species-level calls are incorrect. Therefore, for both species-level and genus-level calls, we recommend that researchers consider reporting classification confidence scores along with results.

We next prepared and sequenced five technical replicates of DNA from a 20 strain mock microbial community and performed taxonomic classification. Even with our limited mock community dataset, we observed hypervariable region-based differences in alpha diversity. Most notably, taxa identified with V9 primers had significantly decreased alpha diversity compared to all other regions across all metrics. V8 results likewise had significantly decreased diversity across most metrics.

We performed six different beta diversity metrics (Bray-Curtis, Jaccard, Canberra, Euclidean, Gower, and Kulczynski) to evaluate differences between hypervariable regions. Distance matrices used in beta diversity analyses are generated from OTU tables, however the OTUs identified were not consistent among hypervariable regions. Therefore, in order to compare results between hypervariable region, we assembled distance matrices using taxonomic results. PCoA analyses demonstrated clustering primarily by hypervariable regions V2, V3, V4, and V6-7. Hypervariable regions V8 and V9 clustered separately from the other regions, again demonstrating the poor performance of amplicon sequencing of these regions in assessing the constituents of the mock community sample.

$$
\text { Consistent with previous reports }(4-6,8) \text {, we found that the taxonomic classification }
$$
results from the mock community samples varied by hypervariable region. Primers targeting the V2, V3, and V6-7 regions identified nearly all the species present in the mock community 
(19/20, 17/20, and 17/20 respectively), V4 identified 16/20 species, V8 identified 15/20 species, and V9 identified only two (2/20) (Fig 3, Table 3). Generally, those regions which identified more species present in the mock community also had more evenly distributed observed taxa (i.e. there were no extreme over- or underestimated taxa which skewed the remaining percent abundances, such as in the case of V9).

The differences in the ability to identify taxa between hypervariable regions could be due to primer bias, database bias, or other biases in the analysis pipeline. Only 21 OTUs were assigned to the V9 region. Of those, two OTUs made up $99.78 \%$ of total V9 reads. Therefore, we deduce that the lack of diversity in the region is likely more related to primer bias than analysis or classification bias. Since V9 lacks sensitivity for many species, we opted to leave this region out of the generalized linear model we used on the clinical samples. V8 also tended to be less sensitive compared to V2, V3, V4, and V6-7, and did not cluster with the other regions on PCoA plots. Therefore, V8 was excluded from further analyses as well.

Researchers can circumvent the issue of choosing only one hypervariable region to analyze by sequencing multiple hypervariable regions in tandem. Since the sensitivity of each hypervariable region for identifying bacterial taxa varies, combining the results from multiple hypervariable regions for analyses may be misleading. Fuks et al. developed Short MUltiple Regions Framework (SMURF), which combines sequences from multiple PCR amplicons in order to provide one overall set of taxonomic profiling results (9). However, this method is computationally intensive and requires proprietary software. Therefore, to utilize information from multiple hypervariable regions at once and to strengthen confidence in the taxonomic abundance results, we incorporated a generalized linear model (GLM) into alpha diversity and 
taxonomic abundance analyses. We excluded V8 and V9 from GLM calculations because of the decreased sensitivity and specificity identified in our mock community analyses.

We demonstrated use of the GLM via analysis of a clinical cohort, where each participant donated two rectal swab samples, one of which was processed fresh and the other one frozen prior to DNA extraction. Alpha diversity analysis revealed increased Evenness in frozen samples compared to fresh samples. This trend was visualized in results from each individual hypervariable region and was strengthened in the GLM. There was no difference in Shannon's diversity, Observed OTUs, and Faith's phylogenetic diversity between fresh and frozen samples. Beta diversity analysis demonstrated clustering of samples by person irrespective of fresh versus frozen status or hypervariable region, with the exception of V9. PERMANOVA analysis confirmed that most of the variation in composition was due to individuals as opposed to storage type. An important limitation of our beta diversity analysis is that in order to compare results from all hypervariable regions in the same analysis, we had to use taxonomic classification as opposed to OTUs. This limits our beta diversity analysis to using only those reads that were assigned taxonomy.

Lastly, we compared taxonomic abundance at multiple levels between fresh and frozen samples and found no taxa at any level had significantly different abundance. This is unsurprising based on our small sample size, the fact that alpha and beta diversity were minimally different between sample type, and the fact that other studies show limited differences between fresh verses frozen samples $(27,28)$. However, Faecalibacterium results highlight the important point that not all regions are able to identify a taxon of interest: V6-7 fails to map any reads to this taxon despite its presence in the sample. Thus, even though the true composition of 
431 a clinical sample may be unknown, examining redundant data from multiple hypervariable

432 regions may help elucidate the true microbial makeup of the sample.

In conclusion, we propose a method to overcome the issues of analyzing multiple

434 amplicons covering multiple hypervariable regions at once. It is critical for the microbiome

435 bioinformatics community to come to a consensus as to the proper way to analyze this type of

436 data in order to maintain data quality, and to be able to compare results across different

437 publications.

$438 \quad$ Funding

This work was supported by The Assistant Secretary of Defense for Health Affairs

440 Endorsed by the Department of Defense through the Prostate Cancer Research Program - Early

441 Investigator Research Award under Award No. W81XWH-18-1-0545 (L.B.P.) and Prostate

442 Cancer Challenge Award 16CHAL13 (K.S.S.). Opinions, interpretations, conclusions and 443 recommendations are those of the author and are not necessarily endorsed by the Department of

444 Defense.

445 Acknowledgements

446 Thank you to the QIIME2 forum community for their help and discussions regarding

447 analyzing multiple hypervariable regions of Ion Torrent data, especially Evan Bolyen, Nicholas

448 Bokulich, Matthew Dillon, Justine Debelius, Colin Brislawn, Jennifer Barb, and Katherine Maki.

449 We also appreciate the help and guidance from Kornel Schuebel, Hai Xu, and Jennifer Meyers of

450 the JHU SKCCC Experimental and Computational Genomics Core; and Bradley Toms and

451 Leonardo Varuzza at ThermoFisher.

452 References 
1. Quince C, Walker AW, Simpson JT, Loman NJ, Segata N. Shotgun metagenomics, from sampling to analysis. Nat Biotechnol. 2017;35(9):833-44.

2. Sanschagrin S, Yergeau E. Next-generation sequencing of 16 S ribosomal RNA gene amplicons. J Vis Exp. 2014(90).

3. Ranjan R, Rani A, Metwally A, McGee HS, Perkins DL. Analysis of the microbiome: Advantages of whole genome shotgun versus $16 \mathrm{~S}$ amplicon sequencing. Biochem Biophys Res Commun. 2016;469(4):967-77.

4. Cai L, Ye L, Tong AH, Lok S, Zhang T. Biased diversity metrics revealed by bacterial 16S pyrotags derived from different primer sets. PLoS One. 2013;8(1):e53649.

5. Barb JJ, Oler AJ, Kim HS, Chalmers N, Wallen GR, Cashion A, et al. Development of an Analysis Pipeline Characterizing Multiple Hypervariable Regions of 16S rRNA Using Mock Samples. PLoS One. 2016;11(2):e0148047.
6. Claesson MJ, Wang Q, O'Sullivan O, Greene-Diniz R, Cole JR, Ross RP, et al. Comparison of two next-generation sequencing technologies for resolving highly complex microbiota composition using tandem variable $16 \mathrm{~S}$ rRNA gene regions. Nucleic Acids Res. 2010;38(22):e200.

7. Pinto AJ, Raskin L. PCR biases distort bacterial and archaeal community structure in pyrosequencing datasets. PLoS One. 2012;7(8):e43093.

8. Tremblay J, Singh K, Fern A, Kirton ES, He S, Woyke T, et al. Primer and platform effects on 16S rRNA tag sequencing. Front Microbiol. 2015;6:771.

9. Fuks G, Elgart M, Amir A, Zeisel A, Turnbaugh PJ, Soen Y, et al. Combining 16S rRNA gene variable regions enables high-resolution microbial community profiling. Microbiome. 2018;6(1):17.

10. Debelius JW, Robeson M, Hugerth LW, Boulund F, Ye W, Engstrand L. A comparison of approaches to scaffolding multiple regions along the $16 \mathrm{~S}$ rRNA gene for improved resolution. bioRxiv. 2021:2021.03.23.436606.

11. Shrestha E, White JR, Yu SH, Kulac I, Ertunc O, De Marzo AM, et al. Profiling the Urinary Microbiome in Men with Positive versus Negative Biopsies for Prostate Cancer. J Urol. 2018;199(1):161-71.

12. Yilmaz P, Parfrey LW, Yarza P, Gerken J, Pruesse E, Quast C, et al. The SILVA and "All-species Living Tree Project (LTP)" taxonomic frameworks. Nucleic Acids Res. 2014;42(Database issue):D643-8.

13. McDonald D, Price MN, Goodrich J, Nawrocki EP, DeSantis TZ, Probst A, et al. An improved Greengenes taxonomy with explicit ranks for ecological and evolutionary analyses of bacteria and archaea. ISME J. 2012;6(3):610-8.

14. Forster SC, Kumar N, Anonye BO, Almeida A, Viciani E, Stares MD, et al. A human gut bacterial genome and culture collection for improved metagenomic analyses. Nat Biotechnol. 2019;37(2):186-92.

15. Bolyen E, Rideout JR, Dillon MR, Bokulich NA, Abnet CC, Al-Ghalith GA, et al. Reproducible, interactive, scalable and extensible microbiome data science using QIIME 2. Nat Biotechnol. 2019;37(8):852-7.

16. Callahan BJ, McMurdie PJ, Rosen MJ, Han AW, Johnson AJ, Holmes SP. DADA2: High-resolution sample inference from Illumina amplicon data. Nat Methods. 2016;13(7):581-3. 17. Good IJ. The population frequencies of species and the estimation of population parameters. Biometrika. 1953;40(3-4):237-64. 
18. Bokulich NA, Dillon MR, Bolyen E, Kaehler BD, Huttley GA, Caporaso JG. q2-sampleclassifier: machine-learning tools for microbiome classification and regression. J Open Res

501 Softw. 2018;3(30).

502 19. Katoh K, Standley DM. MAFFT multiple sequence alignment software version 7:

503 improvements in performance and usability. Mol Biol Evol. 2013;30(4):772-80.

504 20. Price MN, Dehal PS, Arkin AP. FastTree 2--approximately maximum-likelihood trees for 505 large alignments. PLoS One. 2010;5(3):e9490.

506 21. Faith DP, Minchin PR, Belbin L. Compositional dissimilarity as a robust measure of 507 ecological distance. Vegetatio. 1987;69(1-3):57-68.

508 22. Shannon CE. A mathematical theory of communication. ACM SIGMOBILE mobile 509 computing and communications review. 2001;5(1):3-55.

510 23. Jaccard P. Nouvelles recherches sur la distribution florale. Bull Soc Vaud Sci Nat. 511 1908;44:223-70.

512 24. Sorensen TA. A method of establishing groups of equal amplitude in plant sociology

513 based on similarity of species content and its application to analyses of the vegetation on Danish

514 commons. Biol Skar. 1948;5:1-34.

515 25. Lozupone CA, Hamady M, Kelley ST, Knight R. Quantitative and qualitative beta

516 diversity measures lead to different insights into factors that structure microbial communities.

517 Appl Environ Microbiol. 2007;73(5):1576-85.

518 26. Lozupone C, Knight R. UniFrac: a new phylogenetic method for comparing microbial 519 communities. Appl Environ Microbiol. 2005;71(12):8228-35.

520 27. Fouhy F, Deane J, Rea MC, O'Sullivan O, Ross RP, O'Callaghan G, et al. The effects of

521 freezing on faecal microbiota as determined using MiSeq sequencing and culture-based

522 investigations. PLoS One. 2015;10(3):e0119355.

523 28. Bahl MI, Bergstrom A, Licht TR. Freezing fecal samples prior to DNA extraction affects

524 the Firmicutes to Bacteroidetes ratio determined by downstream quantitative PCR analysis.

525 FEMS Microbiol Lett. 2012;329(2):193-7.

526 29. Agnihotry S, Sarangi AN, Aggarwal R. Construction \& assessment of a unified curated

527 reference database for improving the taxonomic classification of bacteria using 16S rRNA

528 sequence data. Indian J Med Res. 2020;151(1):93-103. 


\section{Tables}

532 Table 1. Contents of mock community.

\begin{tabular}{|c|c|c|c|}
\hline Species & $\begin{array}{c}\text { 16S } \\
\text { Copies }^{\mathrm{a}}\end{array}$ & Genus & Family \\
\hline Acinetobacter baumannii & 6 & Acinetobacter & Moraxellaceae \\
\hline Actinomyces odontolyticus & 2 & Actinomyces & Actinomycetaceae \\
\hline Bacillus cereus & 12 & Bacillus & Bacillaceae \\
\hline Bacteroides vulgatus & 7 & Bacteroides & Bacteroidaceae \\
\hline Bifidobacterium adolescentis & 5 & Bifidobacterium & Bifidobacteriaceae \\
\hline Clostridium beijerinckii & 14 & Clostridium & Clostridiaceae \\
\hline Cutibacterium acnes & 4 & Cutibacterium & Propionibacteriaceae \\
\hline Deinococcus radiodurans & 7 & Deinococcus & Deinococcaceae \\
\hline Enterococcus faecalis & 4 & Enterococcus & Enterococcaceae \\
\hline Escherichia coli & 7 & Escherichia & Enterobacteriaceae \\
\hline Helicobacter pylori & 2 & Helicobacter & Helicobacteracae \\
\hline Lactobacillus gasseri & 6 & Lactobacillus & Lactobacillaceae \\
\hline Neisseria meningitidis & 4 & Neisseria & Neisseriaceae \\
\hline Porphyromonas gingivalis & 4 & Porphorymonas & Porphyromonadaceae \\
\hline Pseudomonas aeruginosa & 4 & Psuedomonas & Pseudomonadaceae \\
\hline Rhodobacter sphaeroides & 3 & Rhodobacter & Rhodobacteraceae \\
\hline Staphylococcus aureus & 6 & Staphylococcus & Staphylococcaceae \\
\hline Staphylococcus epidermidis & 5 & Staphylococcus & Staphylococcaceae \\
\hline Streptococcus agalactiae & 7 & Streptococcus & Streptococcaceae \\
\hline Streptococcus mutans & 5 & Streptococcus & Streptococcaceae \\
\hline
\end{tabular}


535 Table 2. Results of in silico analysis.

\begin{tabular}{cccc}
\hline \multicolumn{4}{c}{ Genus Level } \\
\hline Region & False Neg & False Pos & True Pos \\
\hline V2 & $4.85 \%$ & $4.62 \%$ & $90.53 \%$ \\
V3 & $2.82 \%$ & $1.99 \%$ & $95.19 \%$ \\
V4 & $6.22 \%$ & $6.71 \%$ & $87.07 \%$ \\
V6-7 & $5.22 \%$ & $5.79 \%$ & $88.98 \%$ \\
V8 & $6.96 \%$ & $14.50 \%$ & $78.54 \%$ \\
V9 & $21.96 \%$ & $8.14 \%$ & $69.91 \%$ \\
\hline Region & Species Level & True Pos \\
\hline V2 & False Neg & $8.58 \%$ & $77.94 \%$ \\
V3 & $13.48 \%$ & $6.10 \%$ & $77.66 \%$ \\
V4 & $16.24 \%$ & $8.12 \%$ & $66.04 \%$ \\
V6-7 & $25.84 \%$ & $10.03 \%$ & $73.39 \%$ \\
V8 & $16.58 \%$ & $17.46 \%$ & $58.71 \%$ \\
V9 & $23.83 \%$ & $11.56 \%$ & $41.61 \%$ \\
\hline
\end{tabular}

536 Ability of curated database to accurately classify sequences from each hypervariable region from 537 known taxa at the genus and species level in an in silico analysis. 
539 Table 3. Observed species abundance denoted as percent of total.

\begin{tabular}{|c|c|c|c|c|c|c|c|}
\hline Species & Expected & $\mathbf{V} 2$ & $\mathbf{V 3}$ & V4 & V6-7 & V8 & V9 \\
\hline Acinetobacter baumannii & 5.26 & 6.23 & 6.06 & 6.82 & 0.11 & 10.90 & 46.15 \\
\hline $\begin{array}{l}\text { Actinomyces } \\
\text { odontolyticus }\end{array}$ & 1.75 & 1.75 & 0.27 & 1.51 & 0.97 & 1.54 & 0.00 \\
\hline Bacillus cereus & 10.52 & 5.20 & 10.29 & 6.97 & 6.99 & 1.21 & 0.00 \\
\hline Bacteroides vulgatus & 6.14 & 13.11 & 8.19 & 12.48 & 18.57 & 0.00 & 0.00 \\
\hline $\begin{array}{l}\text { Bifidobacterium } \\
\text { adolescentis }\end{array}$ & 4.39 & 5.37 & 0.00 & 4.22 & 0.00 & 5.85 & 0.00 \\
\hline Clostridium beijerinckii & 12.28 & 0.00 & 0.00 & 0.00 & 19.94 & 0.12 & 0.00 \\
\hline Deinococcus radiodurans & 6.14 & 9.88 & 0.00 & 1.47 & 3.29 & 6.31 & 0.00 \\
\hline Enterococcus faecalis & 3.51 & 1.97 & 3.41 & 0.00 & 1.53 & 3.88 & 0.00 \\
\hline Escherichia coli & 6.14 & 5.64 & 6.52 & 4.77 & 8.29 & 0.00 & 53.73 \\
\hline Helicobacter pylori & 1.75 & 5.70 & 2.24 & 2.90 & 2.68 & 0.51 & 0.00 \\
\hline Lactobacillus gasseri & 5.26 & 4.45 & 7.21 & 0.00 & 0.00 & 0.00 & 0.00 \\
\hline Neisseria meningitidis & 3.51 & 5.14 & 4.13 & 6.05 & 0.00 & 9.07 & 0.00 \\
\hline Porphyromonas gingivalis & 3.51 & 2.85 & 5.83 & 7.77 & 8.83 & 0.00 & 0.00 \\
\hline Cutibacterium acnes & 3.51 & 1.35 & 0.27 & 0.17 & 1.97 & 6.04 & 0.00 \\
\hline Pseudomonas aeruginosa & 3.51 & 5.20 & 5.58 & 2.79 & 2.00 & 7.62 & 0.00 \\
\hline Rhodobacter sphaeroides & 2.63 & 3.75 & 4.10 & 3.83 & 2.87 & 12.88 & 0.00 \\
\hline Staphylococcus aureus & 5.26 & 3.19 & 5.44 & 5.71 & 3.00 & 2.08 & 0.00 \\
\hline $\begin{array}{l}\text { Staphylococcus } \\
\text { epidermidis }\end{array}$ & 4.39 & 3.44 & 5.40 & 0.00 & 2.49 & 0.23 & 0.00 \\
\hline Streptococcus agalactiae & 6.14 & 1.89 & 6.31 & 5.46 & 3.06 & 0.00 & 0.00 \\
\hline Streptococcus mutans & 4.39 & 0.87 & 5.47 & 4.81 & 2.09 & 8.03 & 0.00 \\
\hline Total Species Identified & 20 & 19 & 17 & 16 & 17 & 15 & 2 \\
\hline
\end{tabular}




\section{Figures}

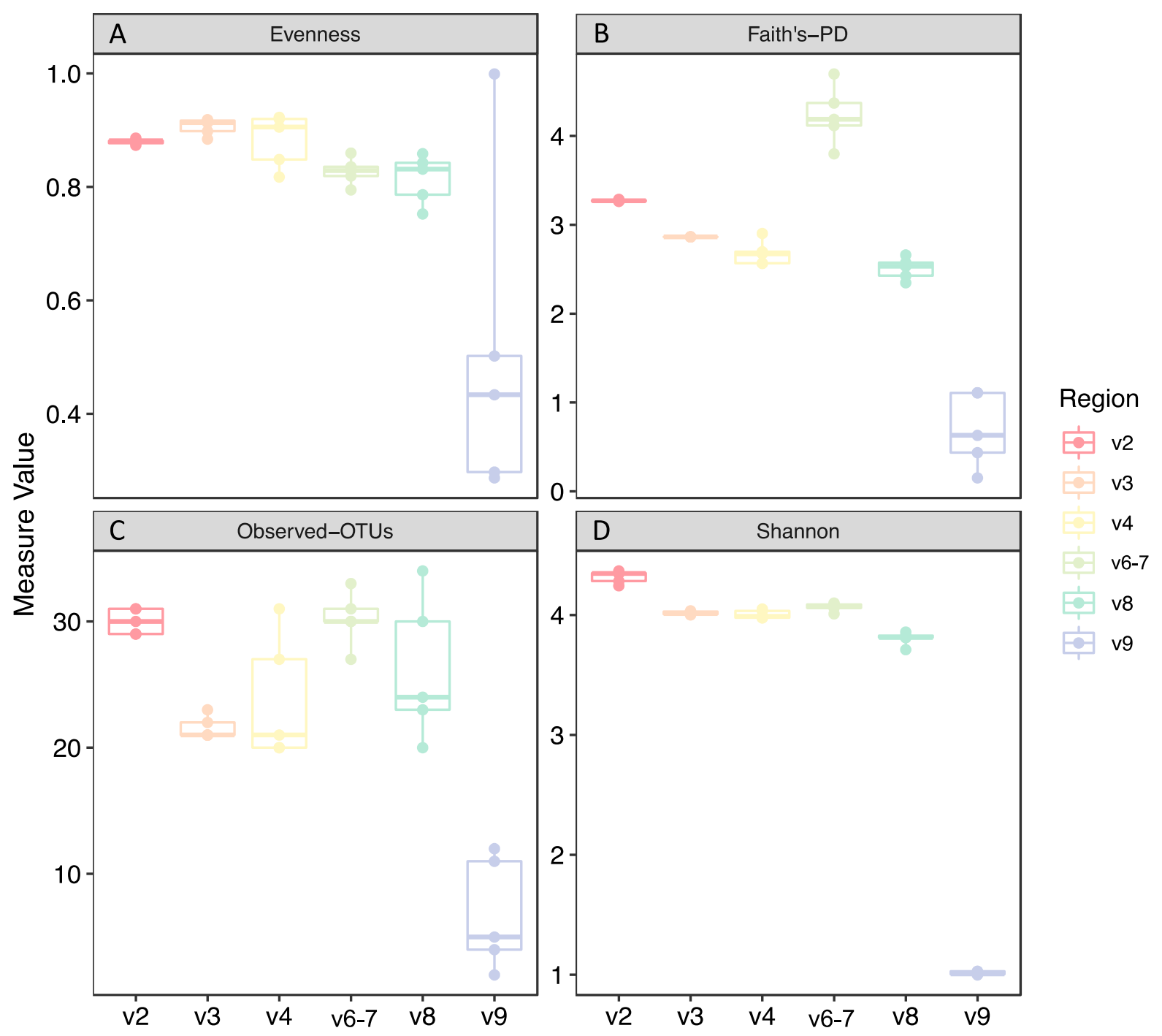

541

542 Fig 1. Alpha diversity analyses of mock community technical replicates by hypervariable

543 region. Evenness (A), Faith's phylogenetic diversity (B), Observed Operational Taxonomic

544 Units (OTUs) (C), Shannon diversity (D). 


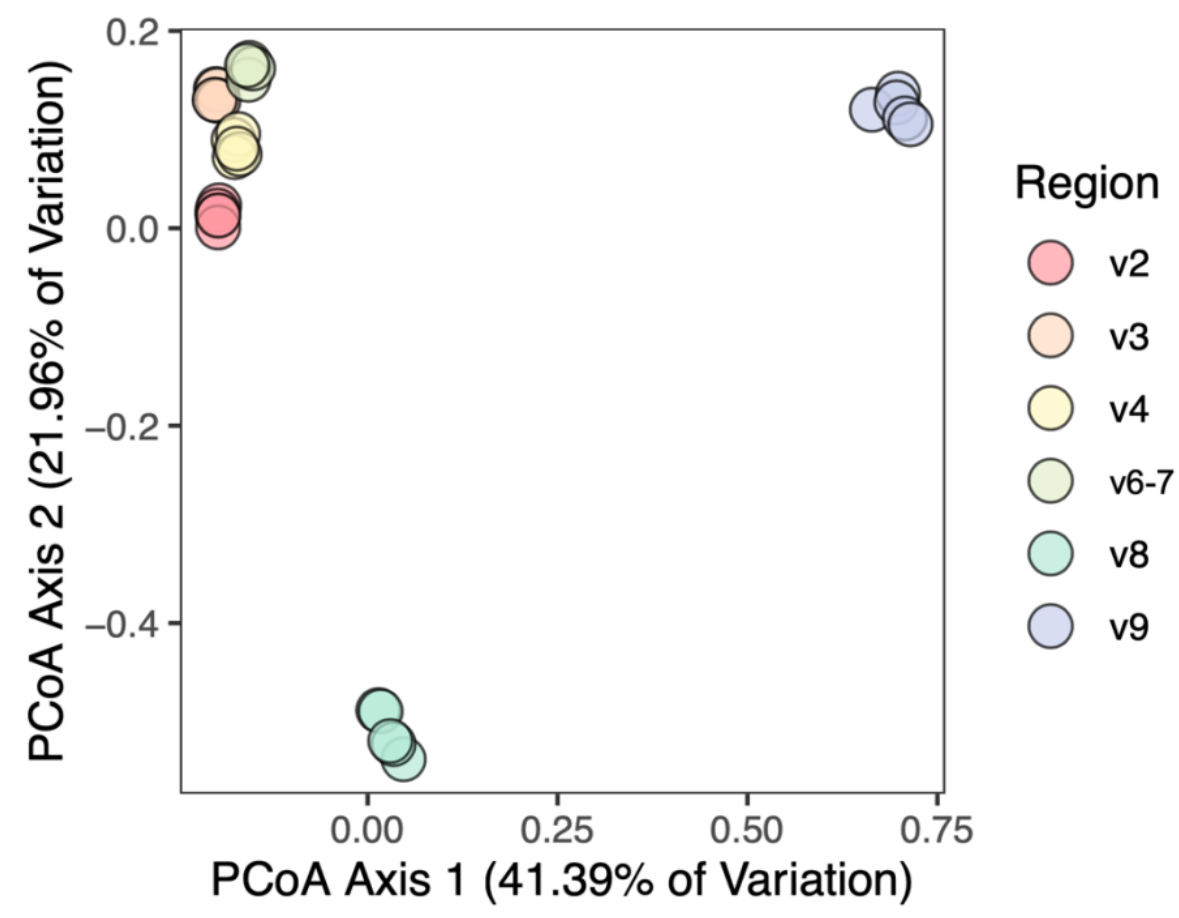

547 Fig 2. Principal coordinates analysis of mock community samples using Canberra distance

548 matrix. 


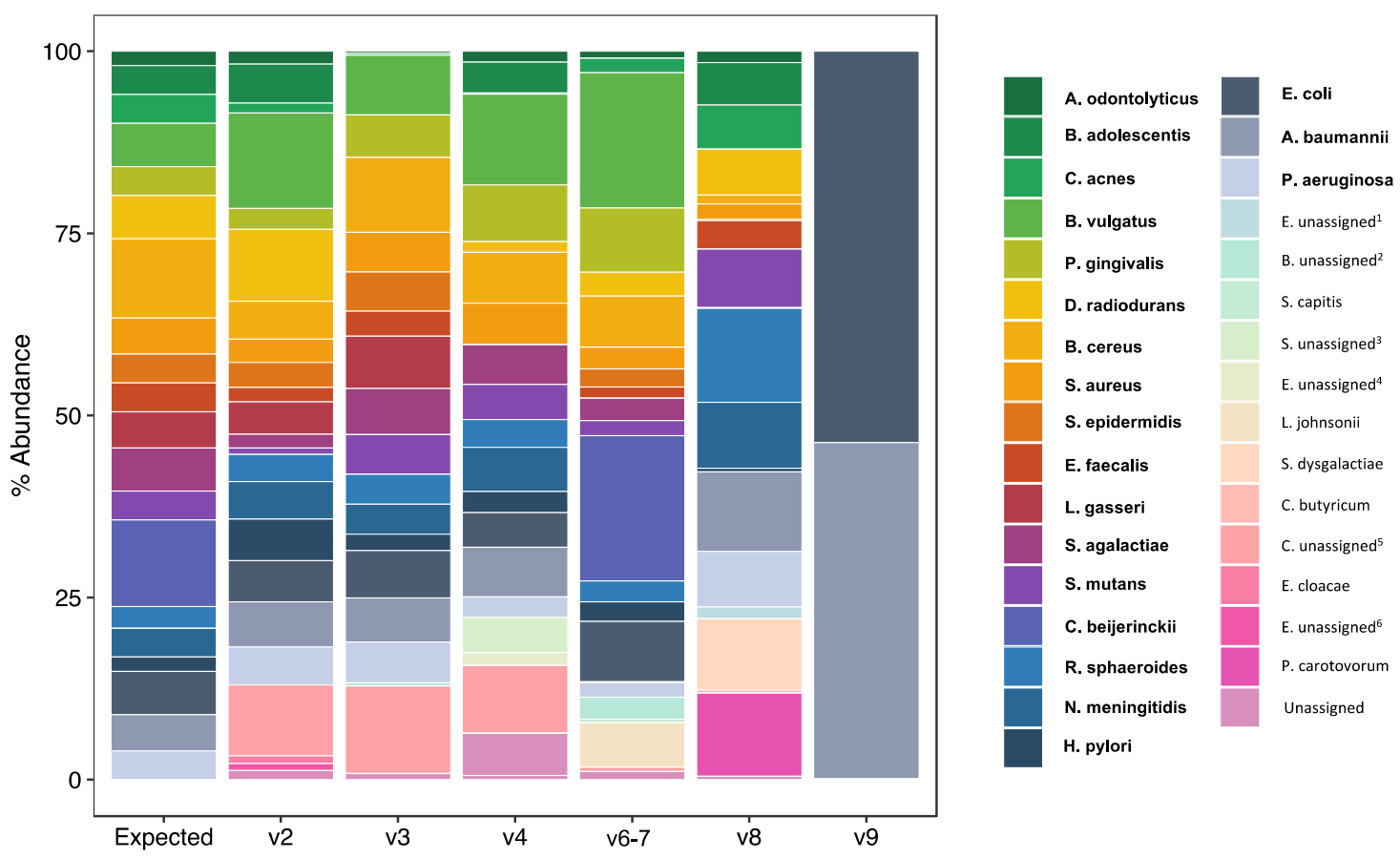

551 Fig 3. Species-level taxonomic barplots of ATCC 20 Strain Mix sequencing results by $16 \mathrm{~S}$

552 rRNA hypervariable region. Bolded taxa are those present in the 20 strain mix.

553 Enterobacteriaceae unassigned ${ }^{1}$, Bifidobacterium unassigned ${ }^{2}$, Staphylococcus unassigned ${ }^{3}$,

554 Enterococcus unassigned ${ }^{4}$, Clostridium sensu stricto 1 unassigned $^{5}$, Enterobacter unassigned ${ }^{6}$. 


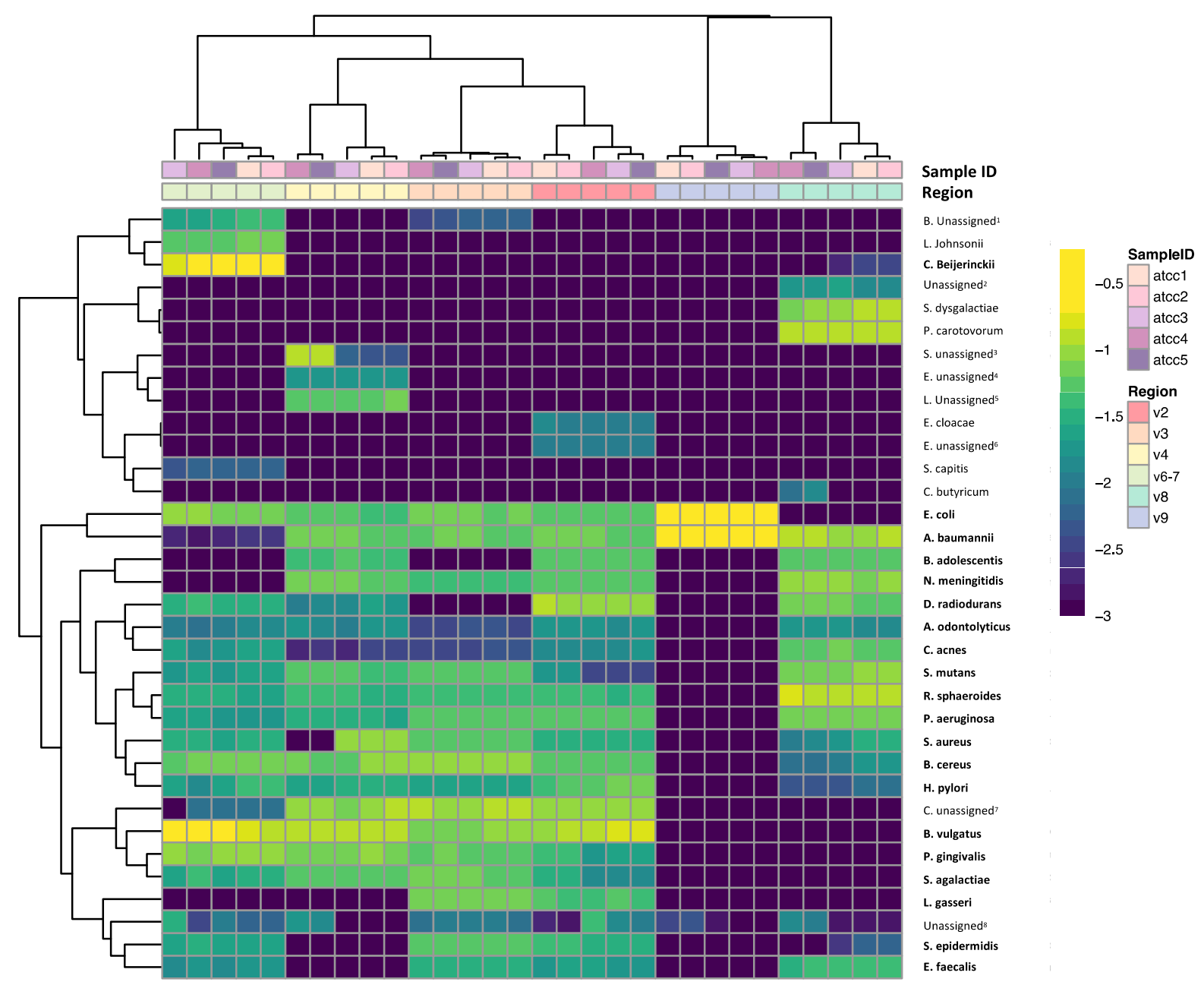

557 those present in the 20 strain mix. Bifidobacterium unassigned ${ }^{1}$, Enterobacteriaceae unassigned ${ }^{2}$,

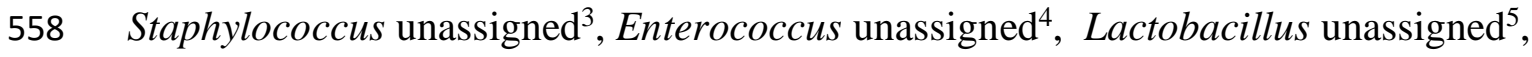

559 Enterobacter unassigned ${ }^{6}$, Clostridium sensu stricto 1 unassigned $^{7}$, Unassigned at every

taxonomic level ${ }^{8}$. 

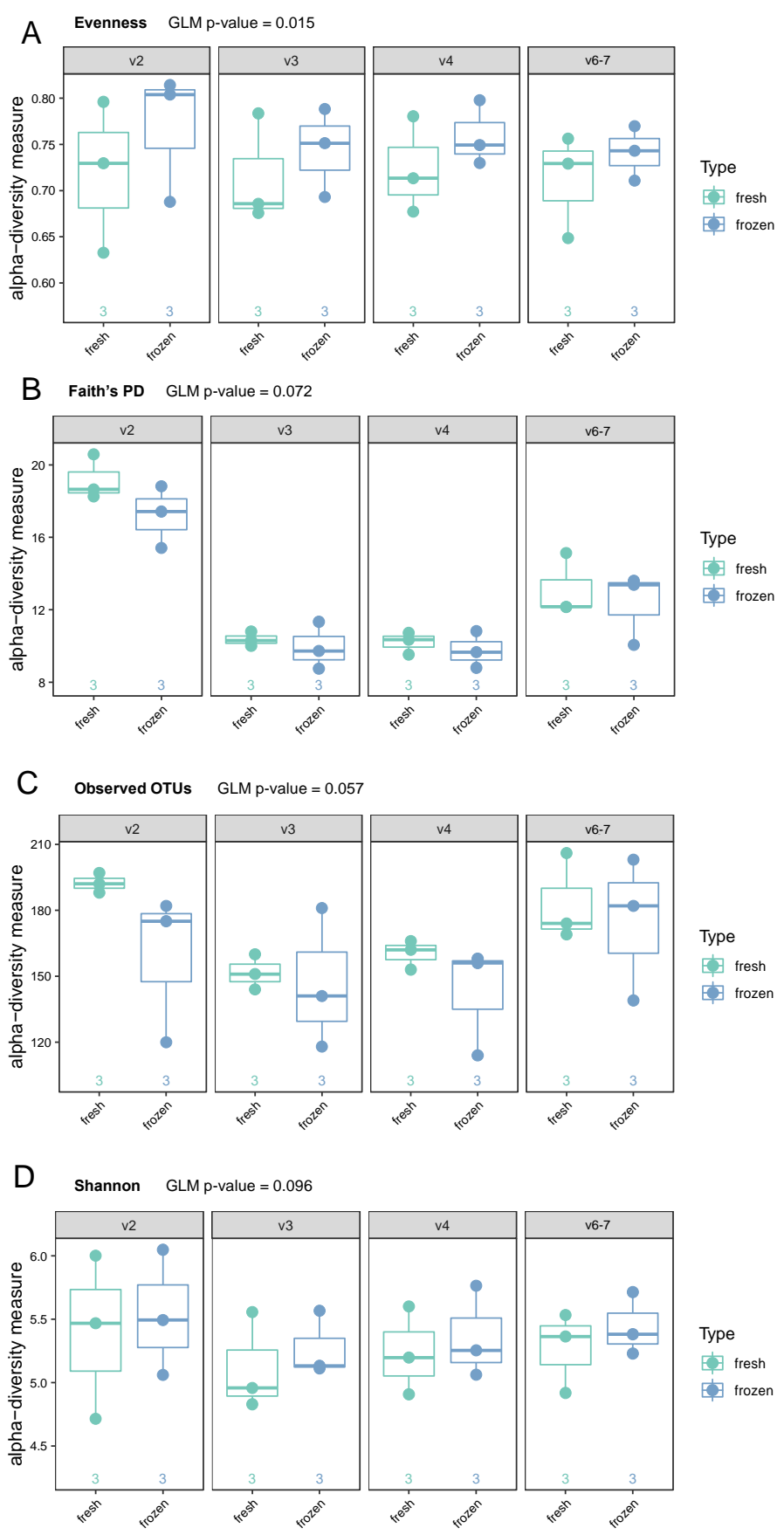

562 Fig 5. Alpha diversity analyses of six clinical samples by type (fresh or frozen) and

563 hypervariable region. Each patient provided two swabs, one of which was frozen prior to DNA

564 extraction. Evenness (A), Faith's phylogenetic diversity (B), Observed Operational Taxonomic

565 Units (OTUs) (C), Shannon diversity (D). 


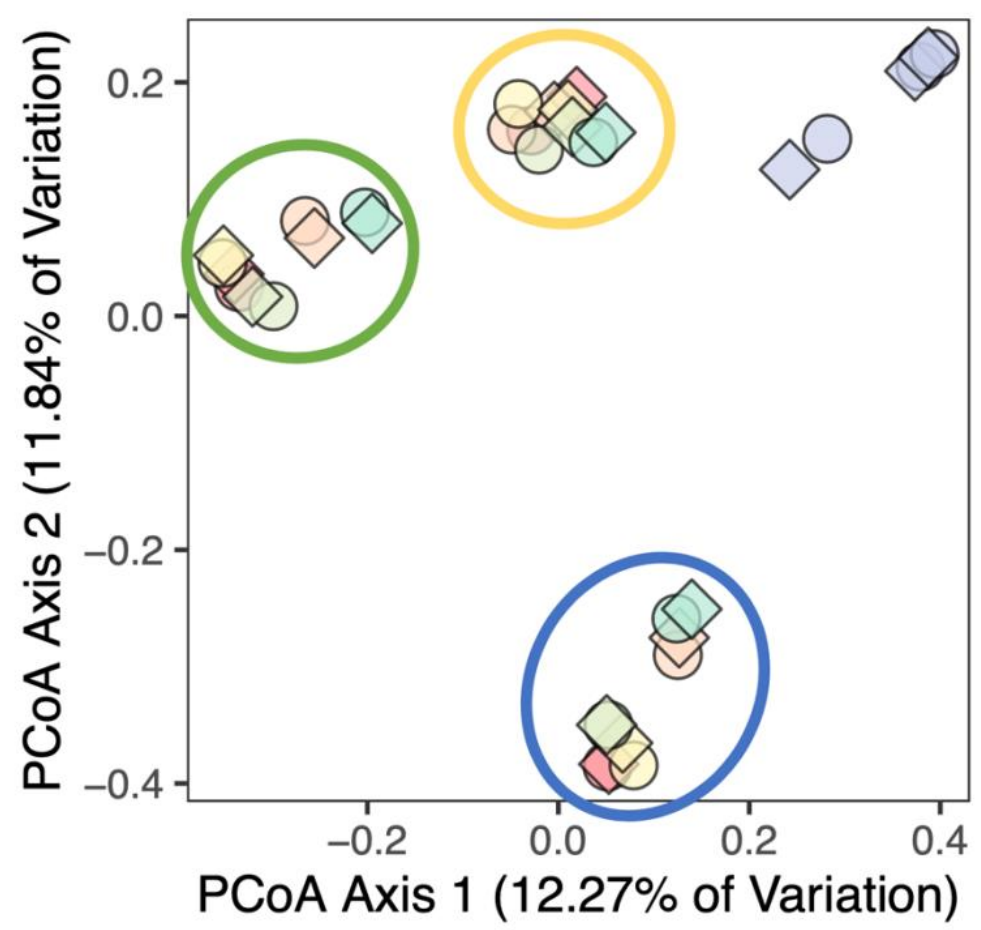

\section{Region}

○2

○3

$\bigcirc \mathrm{v} 4$

○ v6-7

○ v8

○ v9

Type

fresh

$\diamond$ frozen

566

567 Fig 6. Principal coordinates analysis of clinical cohort using Canberra distance matrix.

568 Samples and regions from the same person are circled, excluding V9. Results cluster by

569 individual and by V9 region (not circled), but not by fresh versus frozen status. 
A Bacteroidetes, $p=0.65$
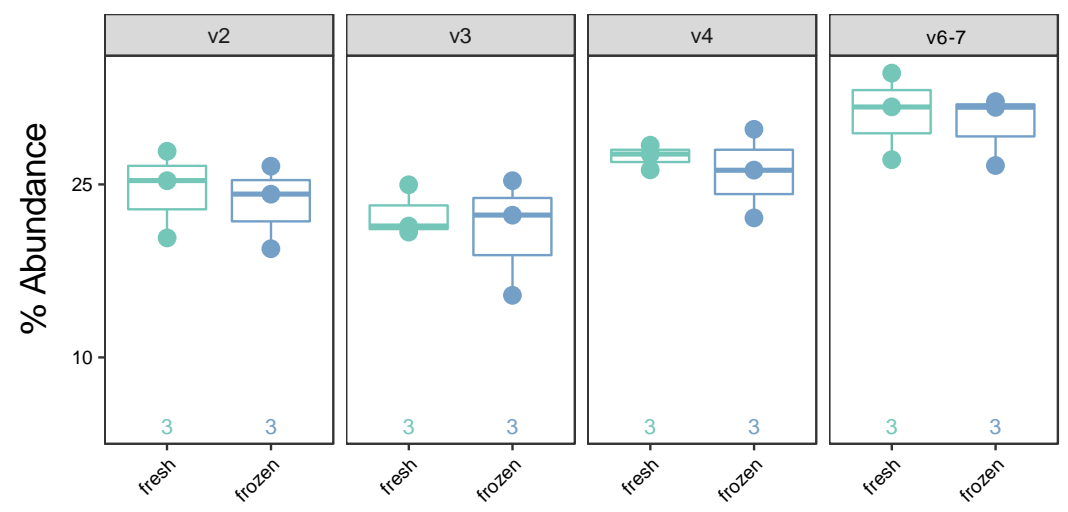

Type

E fresh

宁 frozen

B Firmicutes, $p=0.93$
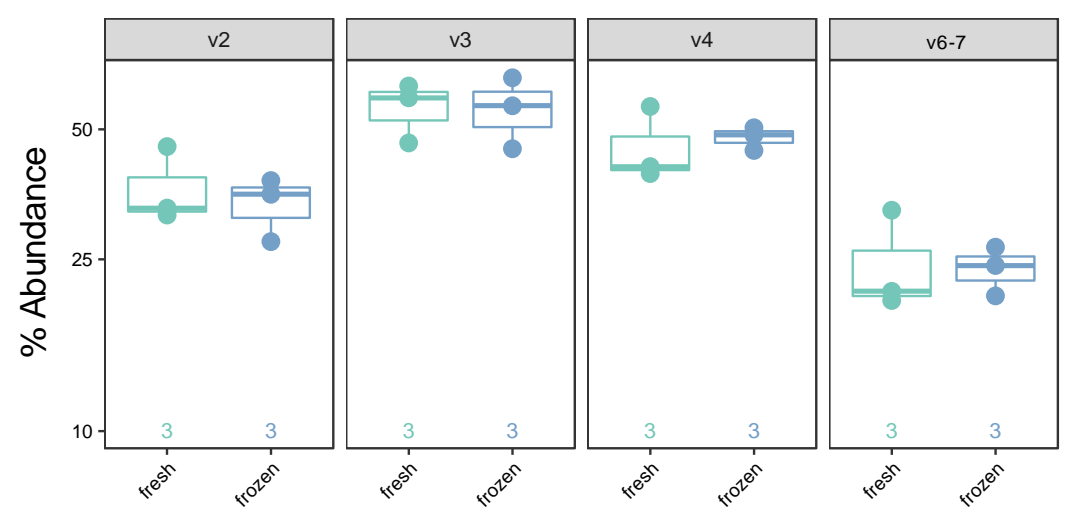

Type

$\emptyset$ fresh

安 frozen

C Faecalibacterium, $\mathrm{p}=0.99$
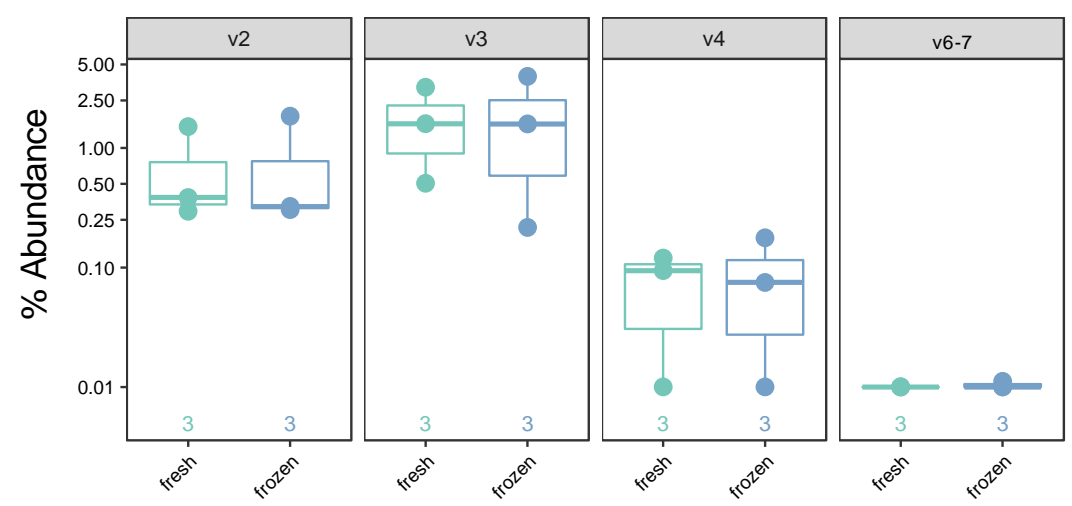

Type

畐 fresh

Ð frozen

572 Fig 7. Percent abundance of Bacteroidetes, Firmicutes, and Faecalibacterium by sample

573 type (fresh vs. frozen) and hypervariable region. P-value was calculated with a log-

574 transformed GLM, and is false discovery rate-adjusted. 


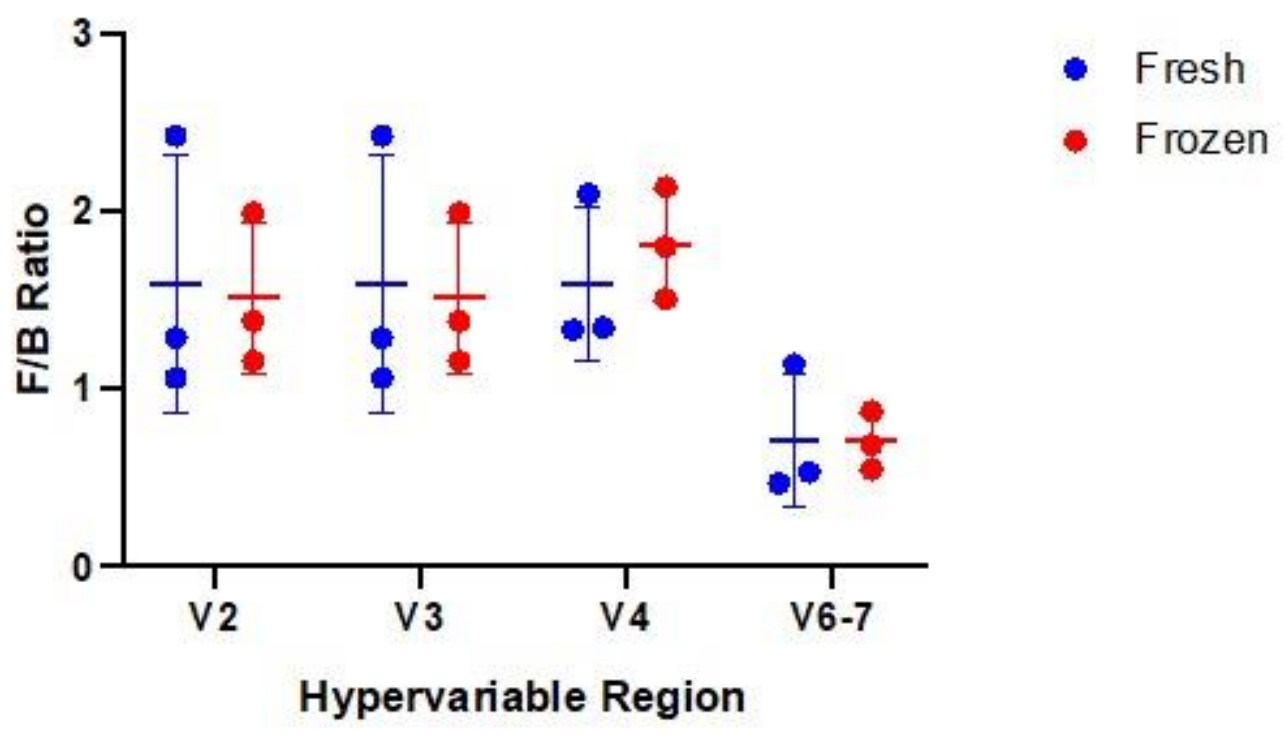

576

577 Fig 8. Comparison of Firmicutes to Bacteroidetes (F/B) ratio in fresh versus frozen samples

578 by hypervariable region.

579

580 Supporting information

581 S1 Fig. Other beta diversity metrics (Bray-Curtis, Jaccard, Euclidean, Gower, and

582 Kulczynski), mock samples

583 S1 Table. Table of contaminants.

584 S1 File. In silico taxonomic validation results.

585 S2 File. Alpha diversity statistics.

586 S3 File. Filtered percent abundance.

587 S4 File. PERMANOVA analysis of fresh vs frozen clinical samples. 